\title{
Early pregnancy factor, chaperonin 10 and rheumatoid arthritis; the story unravels
}

\author{
Thiago DC Hirata ${ }^{1,2}$, Helder Nakaya ${ }^{1}$, Thuy TT Le ${ }^{2}$, Yee S Poo $^{2}$ and Andreas Suhrbier ${ }^{2 *}$ \\ ${ }^{1}$ School of Pharmaceutical Sciences, University of Sao Paulo, Sao Paulo, Brazil \\ ${ }^{2}$ QIMR Berghofer Medical Research Institute, Brisbane, Queensland 4029, Australia
}

Treatment with recombinant chaperonin 10 (rCpn10) was shown to be efficacious in rheumatoid arthritis patients. Chaperonin 10 (Cpn10) is believed to represent the immunosuppressive Early Pregnancy Factor (EPF) previously identified in pregnancy serum. Herein we argue that rCpn10's immunosuppressive activity was likely due to endotoxin tolerance. Furthermore, Cpn10 is not elevated in pregnancy serum, making it a poor candidate for EPF. Although the Cpn10/EPF story provided an enticing rationale, the evidence that Cpn10 is EPF, and that Cpn10 has anti-inflammatory activity is far from compelling.

Early pregnancy factor (EPF) was originally described in a 1977 Lancet paper as an immunosuppressive factor present in human pregnancy serum that could be detected by a rosette inhibition test (RIT) [1]. Lymphocytes form spontaneous rosettes with sheep red blood cells via CD2, an activity that can be inhibited by anti-lymphocyte anti-serum or anti-CD2 antibodies [2]. Incubation of lymphocytes with pregnancy serum (but not normal serum) significantly reduced the concentration of anti-lymphocyte anti-serum required to inhibit rosette formation [1]. EPF might more accurately be described as a RIT activity, as no actual factor was identified in this study [1]. The activity was identified in pregnancy serum from other mammals, including cows, horses, pigs and mice, albeit with consistency issues [3]. In humans RIT activity was present until week 28 of pregnancy, but was back to baseline at 31 weeks [4-6]. Using the RIT assay, EPF was subsequently professed to be heat shock protein 10 (Hsp10), also known as chaperonin 10 (Cpn10) [7,8].

Buoyed by the observation that rheumatoid arthritis is often ameliorated during pregnancy [9], recombinant Cpn10 (rCpn10) was subsequently developed by CBio Ltd. as an immunomodulatory biologic (XToll) [10]. A randomised, double-blind, multi-centre human trial supported the view that $\mathrm{rCpn} 10$ (5-10 mg intravenously twice a week) was efficacious for treating symptoms of rheumatoid arthritis (RA). The trial results were published in Lancet [11]. Efficacy was subsequently reported for rCpn10 in the treatment of multiple sclerosis [12] and psoriasis [13], with a trial for lupus also undertaken (NIH USA ClinicalTrials NCT01838694).

However, after publication of the RA trial results [11], some reservations were raised. A comment published in Lancet argued that endotoxin tolerance may have played a role; $\mathrm{rCpn} 10$ was produced in bacteria and may thus have been contaminated with low levels of endotoxin [14]. Endotoxin tolerance is a well described phenomenon whereby repeated exposure to endotoxin results in anti-inflammatory/ immunosuppressive immunomodulation $[15,16]$.

De-identified microarray, DAS28 and pharmacokinetic data from a small number of patients enrolled in the RA trial [11] was made available by Invion Ltd., who purchased CBio and are not pursuing development of XToll. A mean Cmax for rCpn10 of $1820+$ SD 115 $\mathrm{ng} / \mathrm{ml}$ and $\mathrm{T}_{1 / 2}$ of $1+\mathrm{SD} 0.11$ hour was determined in a subgroup of patients $(n=3)$ enrolled in the RA trial. PBMC were obtained at baseline (T0) and after 56 days of intravenous treatment with $10 \mathrm{mg}$ of rCpn10 twice a week (T56). Paired (T0/T56) microarray data from PBMC from 4 patients (GSE112809) passed quality control assessments. The DAS28 score improvement in each of these 4 patients over this period (5.67 to $2.81,5.58$ to $4.4,4.83$ to 3.54 , and 6.3 to 5.58 ; paired t test $\mathrm{p}=0.048$ ) was consistent with [11]. Differentially expressed genes (DEGs) were identified using a paired comparison for T56 verses T0 for each of the 4 patients. The analysis identified 328 DEGs that were up-regulated and 139 DEGs that were down-regulated at T56 (using a $\mathrm{p}<0.05$ cut-off) (Supplementary File). Using Gene Set Enrichment Analyses (fGSEA), the up-regulated DEGs were compared with publicly available transcriptional profiles from (i) human peripheral blood treated with LPS [17] and (ii) human peripheral blood treated with monophosphoryl lipid A (MPLA) [17], which is a Toll-like receptor 4 (like LPS) and a Toll-like receptor 2 agonist. Significant enrichments were observed (i) LPS, NES $=1.31, \mathrm{FDR}=0.037$ and (ii) MPLA, NES $=1.43, \mathrm{FDR}=0.0038$ (Figure 1a). Analysis of down-regulated genes using Ingenuity upstream regulator analysis identified the immunosuppressive cytokines IL$10(\mathrm{p}=0.043)$ and TGF $\beta 1(\mathrm{p}=0.045)$ as potentially responsible for down-regulation of several genes (Figure 1b), consistent with [11], the DAS28 score improvements, and also endotoxin tolerance [16]. As patient numbers were low and low stringency cut-offs were used, these results might be viewed as indicative only, but they are consistent with induction of endotoxin tolerance by rCpn10 (as suggested [14]). Endotoxin contamination of recombinant proteins is hardly new, although bioassays or murine systems that reliably predict endotoxin tolerance (e.g. from repeated low dose endotoxin exposure) are arguably not well developed $[18,19]$. Humans are as much as 100,000 fold more sensitive to LPS than mice [20].

The molecular basis for RIT activity in pregnancy sera is unclear, as is the selectivity and specificity of the RIT assay. We thus surveyed the proteomics literature to ascertain whether Cpn10 (or Hsp10) was actually present in human pregnancy serum. In 6 studies [21-25] only one study identified one Hsp10 peptide [26], although 100-800 other proteins were identified in these studies. Hsp10 is not intrinsically

Correspondence to: Andreas Suhrbier, QIMR Berghofer Medical Research Institute, Brisbane, Queensland 4029, Australia, E-mail: Andreas.Suhrbier@ qimrberghofer.edu.au

Received: March 14, 2018; Accepted: March 30, 2018; Published: April 03, 2018 
a

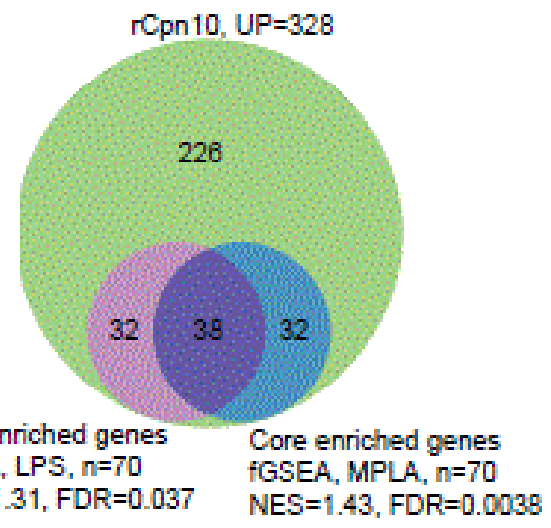

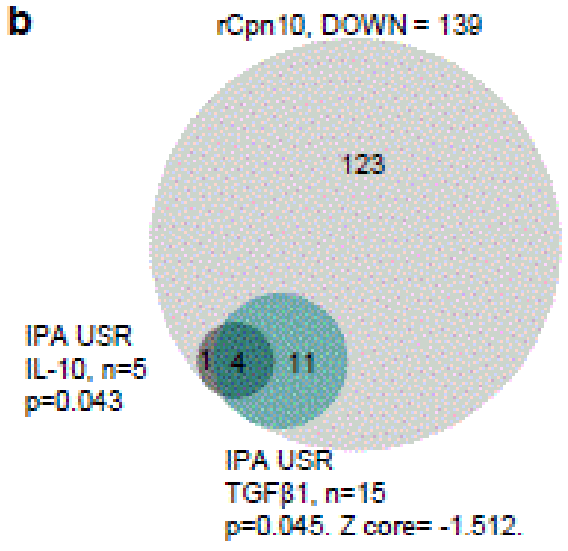

Figure 1. PBMC-derived RNA from 4 rheumatoid arthritis patients treated with rCpn10 was subjected to gene expression microarray analysis using the Affymetrix Human Genome U133 Plus 2.0 Array platform. Microarray data was converted into expression measures and normalised with robust multiarray average (RMA) and gene ID conversion by affy Bioconductor package. The quality control was performed using arrayqualitymetrics Bioconductor package. The paired t-test based on the limma Bioconductor package was used to identify differentially expressed genes (DEGs) between T0 (before rCpn10 treatment initiation) and T56 (after 56 days of rCpn10 treatment) (p $<0.05$ and fold change $>1.0$ ). (a) Up-regulated DEGs (after rCpn10 treatment) $(\mathrm{n}=328)$ were used in pre-Ranked Gene Set Enrichment Analyses. These were performed using fGSEA (R) and pre-ranked gene expression fold change data from microarrays of human blood treated for 90 mins with LPS or MPLA (GSE72557). Of the 328 rCpn10 up-regulated genes, 295 were present in the two data sets. Of these, 102 (32+38+32) were identified as core enriched genes by fGSEA for LPS and/or MPLA stimulation. (b) Down-regulated genes (after rCpn10 treatment) were analysed by Ingenuity Pathway Analysis (IPA) upstream regulator function (direct and indirect). A small but significant IL-10 and TGF $\beta 1$ signature was identified (numbers of "Target molecules in dataset" from IPA output are indicated).

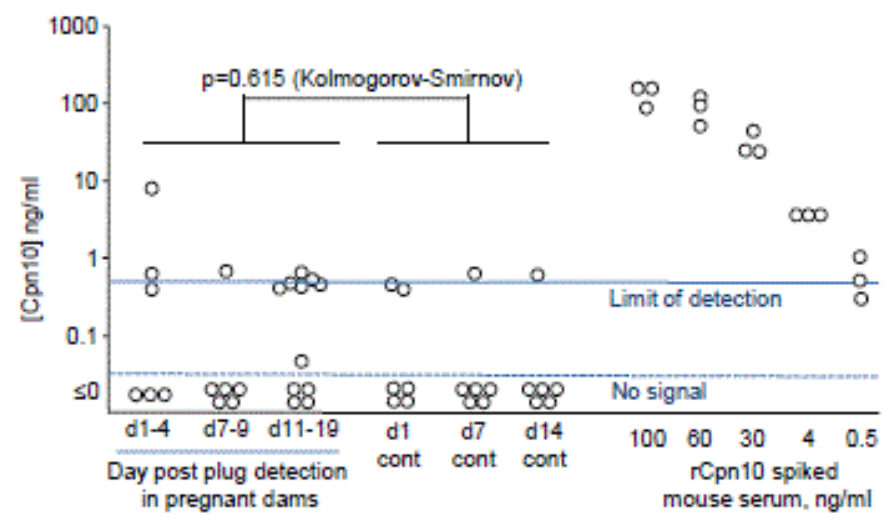

Figure 2. Cpn10 levels in mouse pregnancy sera. Sera obtained from pregnant Balb/c mice and non pregnant mice (cont) at the indicated times was analysed for Cpn10 concentration in duplicate using a capture ELISA.

difficult to detect by mass spectroscopy $[27,28]$. Using an antibodycapture ELISA (detection limit $0.5 \mathrm{ng} / \mathrm{ml}$ ) [29], Cpn10 levels were mostly at or below the detection limit, with no indication that levels in mouse pregnancy were significantly elevated (Figure 2). Cpn10 has been shown to circulate in non-pregnant humans (mean $6.5+\mathrm{SE} 5 \mathrm{ng} / \mathrm{ml}$, range $0-115, n=23$ ) [29]. Good evidence that Cpn10 is elevated during pregnancy and therefore a candidate for EPF is thus lacking. Finally, other factors that mediate maternal tolerance have been identified [30], although a broad spectrum of factors are likely involved [31].

In summary, the evidence that Cpn10 is EPF, and that Cpn10 has anti-inflammatory activity is not compelling. The EPF/Cpn10 story continues to inspire [32,33], but clearly needs to be carefully re-examined.

\section{References}

1. Morton H, Rolfe B, Clunie GJ (1977) An early pregnancy factor detected in human serum by the rosette inhibition test. Lancet 1: 394-397.

2. Yoshioka K, Iwamura S, Kamomae H (1995) Application of anti-bovine CD2 monoclonal antibody to the rosette inhibition test for detection of early pregnancy factor in cattle. $J$ Vet Med Sci 57: 721-725.
3. Koch E, Ellendorff F (1985) Prospects and limitations of the rosette inhibition test to detect activity of early pregnancy factor in the pig. $J$ Reprod Fertil 74: 29-38. [Crossref]

4. Cheng SJ, Zheng ZQ (2004) Early pregnancy factor in cervical mucus of pregnant women. Am J Reprod Immunol 51: 102-105.

5. Wang HN, Zheng ZQ (1990) Detection of early pregnancy factor in fetal sera. Am J Reprod Immunol 23: 69-72. [Crossref]

6. Smart YC, Roberts TK, Fraser IS, Cripps AW, Clancy RL (1982) Validation of the rosette inhibition test for the detection of early pregnancy in women. Fertil Steril 37: 779-785.

7. Cavanagh AC, Morton $\mathrm{H}$ (1994) The purification of early-pregnancy factor to homogeneity from human platelets and identification as chaperonin 10. Eur J Biochem 222: 551-560.

8. Morton H (1998) Early pregnancy factor: an extracellular chaperonin 10 homologue. Immunol Cell Biol 76: 483-496. [Crossref]

9. Goin DE, Smed MK, Pachter L, Purdom E, Nelson JL, et al. (2017) Pregnancyinduced gene expression changes in vivo among women with rheumatoid arthritis: a pilot study. Arthritis Res Ther 19: 104

10. van Eden W (2008) XToll, a recombinant chaperonin 10 as an anti-inflammatory immunomodulator. Curr Opin Investig Drugs 9: 523-533.

11. Vanags D, Williams B, Johnson B, Hall S, Nash P, et al. (2006) Therapeutic efficacy and safety of chaperonin 10 in patients with rheumatoid arthritis: a double-blind randomised trial. Lancet 368: 855-863.

12. Broadley SA, Vanags D, Williams B, Johnson B, Feeney D, et al. (2009) Results of a phase IIa clinical trial of an anti-inflammatory molecule, chaperonin 10, in multiple sclerosis. Mult Scler 15: 329-336.

13. Williams B, Vanags D, Hall S, McCormack C, Foley P, et al. (2008) Efficacy and safety of chaperonin 10 in patients with moderate to severe plaque psoriasis: evidence of utility beyond a single indication. Arch Dermatol 144: 683-685.

14. Focosi D (2006) Chaperonin 10 for rheumatoid arthritis. Lancet 368: 1961. [Crossref]

15. Pena OM, Pistolic J, Raj D, Fjell CD, Hancock RE (2011) Endotoxin tolerance represents a distinctive state of alternative polarization (M2) in human mononuclear cells. J Immunol 186: 7243-7254.

16. Biswas SK, Lopez-Collazo E (2009) Endotoxin tolerance: new mechanisms, molecules and clinical significance. Trends Immunol 30: 475-487.

17. Enkhbaatar P, Nelson C, Salsbury JR, Carmical JR, Torres KE, et al. (2015) Comparison of gene expression by sheep and human blood stimulated with the TLR 4 agonists lipopolysaccharide and monophosphoryl lipid A. PLoS One 10: e0144345. [Crossref] 
18. Johnson BJ, Le TT, Dobbin CA, Banovic T, Howard CB, et al. (2005) Heat shock protein 10 inhibits lipopolysaccharide-induced inflammatory mediator production. $J$ Biol Chem 280: 4037-4047.

19. Kulkarni OP, Ryu M, Kantner C, Sardy M, Naylor D, et al. (2012) Recombinant chaperonin 10 suppresses cutaneous lupus and lupus nephritis in MRL-(Fas)lpr mice. Nephrol Dial Transplant 27: 1358-1367.

20. Munford RS (2010) Murine responses to endotoxin: another dirty little secret? J Infect Dis 201: 175-177.

21. Kim SM, Cho BK, Kang MJ, Norwitz ER, Lee SM, et al. (2016) Expression changes of proteins associated with the development of preeclampsia in maternal plasma: A casecontrol study. Proteomics 16: 1581-1589.

22. Blankley RT, Fisher C, Westwood M, North R, Baker PN, et al. (2013) A labelfree selected reaction monitoring workflow identifies a subset of pregnancy specific glycoproteins as potential predictive markers of early-onset pre-eclampsia. Mol Cell Proteomics 12: 3148-3159.

23. Zhang Y, Kang Y, Zhou Q, Zhou J, Wang H, et al. (2014) Quantitative proteomic analysis of serum from pregnant women carrying a fetus with conotruncal heart defect using isobaric tags for relative and absolute quantitation (iTRAQ) labeling. PLoS One 9: e111645. [Crossref]

24. Park J, Cha DH, Lee SJ, Kim YN, Kim YH, Kim KP (2011) Discovery of the serum biomarker proteins in severe preeclampsia by proteomic analysis. Exp Mol Med 43: 427-435.

25. Lopez Uriarte GA, Burciaga Flores CH, Torres de la Cruz VM, Medina Aguado MM, Gomez Puente VM, et al. (2017) Proteomic profile of serum of pregnant women carring a fetus with Down syndrome using nano uplc Q-tof ms/ms technology. J Matern Fetal Neonatal Med 1-7.
26. Zhao C, Wang F, Wang P, Ding H, Huang X, Shi Z (2015) Early second-trimester plasma protein profiling using multiplexed isobaric tandem mass tag (TMT) labeling predicts gestational diabetes mellitus. Acta Diabetol 52: 1103-1112.

27. Xin L, Xu B, Ma L, Hou Q, Ye M, et al. (2016) Proteomics study reveals that the dysregulation of focal adhesion and ribosome contribute to early pregnancy loss. Proteomics Clin Appl 10: 554-563.

28. Melle C, Bogumil R, Ernst G, Schimmel B, Bleul A, von Eggeling F (2006) Detection and identification of heat shock protein 10 as a biomarker in colorectal cancer by protein profiling. Proteomics 6: 2600-2608.

29. Anraku I, Rajasuriar R, Dobbin C, Brown R, Lewin SR, et al. (2012) Circulating hea shock protein 60 levels are elevated in HIV patients and are reduced by anti-retroviral therapy. PLoS One 7: e45291. [Crossref]

30. Schumacher A (2017) Human chorionic gonadotropin as a pivotal endocrine immune regulator initiating and preserving fetal tolerance. Int J Mol Sci 18. [Crossref]

31. Bonney EA (2017) Alternative theories: Pregnancy and immune tolerance. J Reprod Immunol 123: 65-71. [Crossref]

32. Chen Q, Zhu X, Chen R, Liu J, Liu P, et al. (2016) Early pregnancy factor enhances the generation and function of CD4+CD25+ regulatory T cells. Tohoku J Exp Med 240: 215-220. [Crossref]

33. Fan W, Fan SS, Feng J, Xiao D, Fan S, et al. (2017) Elevated expression of HSP10 protein inhibits apoptosis and associates with poor prognosis of astrocytoma. PLoS One 12: e0185563. [Crossref]

Copyright: (C2018 Hirata TDC. This is an open-access article distributed under the terms of the Creative Commons Attribution License, which permits unrestricted use, distribution, and reproduction in any medium, provided the original author and source are credited. 